\title{
Weighted window method for TCP fairness in WLANs
}

\begin{abstract}
Distributed Coordination Function (DCF) in IEEE 802.11 WLANs cannot guarantee perstation TCP fairness among wireless stations. In order to satisfy users, and guarantee to allocate fair bandwidth among wireless users regardless of the number and direction of flows, the Weighted Window method is proposed. This method can be simply implemented in mobile stations without having to modify the MAC layer. In this paper, the proposed method and its properties will be presented in detail using ns 2 simulation tool under various network conditions. The simulation results are shown that the proposed method achieves fair throughput among stations as compared with previous methods.
\end{abstract}

